Check for updates

Cite this: RSC Adv., 2018, 8, 11119

\title{
Mesogen-co-polymerized transparent polyimide as a liquid-crystal alignment layer with enhanced anchoring energy $\dagger$
}

\author{
Faqin Tong, ${ }^{a}$ Shuangshuang Chen, ${ }^{\mathrm{b}}$ Zhao Chen, ${ }^{\mathrm{a}}$ Xuemin Lu*a and Qinghua Lu (D) *a
}

Enhancing the azimuthal anchoring energy of the aligning film is a key requirement to improve the quality of liquid-crystal displays. Based on the assumption of mesogenic side groups being able to align liquid-crystal (LC) molecules, we have designed a novel transparent polyimide bearing cyanobiphenyl mesogenic side chains, which has a similar structure to that of liquid crystal 5CB. A pyrimidyl diamine monomer with a cyanobiphenyl mesogenic unit was first synthesized, and then co-polymerized with 4,4'diaminodiphenyl ether and 4,4'-oxydiphthalic anhydride. The obtained polyimide (PI) exhibited good thermal stability and mechanical properties, as well as high optical transparency with transmittance of up to $86 \%$ in the wavelength range $450-700 \mathrm{~nm}$. By evaluating the adhesion force between PI films and LCs with a super-sensitive microelectro-mechanical balance system, we have verified that appending mesogenic groups on the side chains of PI strengthens intermolecular interactions between the LC and PI surfaces, thereby enhancing the surface anchoring energy. Compared with the unmodified Pl, the anchoring energy of the derivatized PI was improved more than 2.2-fold. By virtue of its good transparency and strong anchoring energy, this PI would seem to be an ideal candidate as a LC alignment film, and the concept of mesogens aligning LCS may open a new door in the design of LC alignment films.

Received 17th January 2018

Accepted 13th March 2018

DOI: $10.1039 / c 8 r a 00479 j$

rsc.li/rsc-advances parameters, namely the pretilt angle of the LC molecules and the surface azimuthal anchoring energy. ${ }^{12}$ The LCDs according to the pretilt angle is divided into two categories: zero and nonzero pretilt angle LCDs. Zero pretilt angle LCDs includes inplane switching (IPS) and fringe-field switching (FFS) LCD modes. Nonzero pretilt angle LCDs includes twisted nematic displays (TN-LCD): $1-3^{\circ}$; super twisted nematic displays (STNLCD): $5-30^{\circ}$; no-bias optically-compensated bend (OCB) LCD and the bi-stable bend-splay (BBS) LCD: $45-60^{\circ}$; multi-domain vertical alignment LCD: $\sim 90^{\circ}$. Strong azimuthal anchoring energy is needed for both zero and nonzero pretilt angle LCDs, what's more, a proper pretilt angle is required for TN-LCDs to prevent the formation of reverse tilt domains, which will reduce contrast and form speckle in the displayed image due to different refractive index in the small reverse tilt domains. The pretilt angle of LC molecules can be modulated for actual applications. However, obtaining an alignment layer with high anchoring energy is still a challenge for the development of LCDs. Strong intermolecular interaction between LC molecules and polymer chains is desired, so as to enhance the aligning ability without any damage to the polymer film. To achieve this, reactive mesogens have been used to improve the azimuthal anchoring energies of polyimide (PI) alignment layers in three ways. Firstly, the mesogen may be pre-added to the LC solution to form a reactive mesogen network on the surface of the alignment layer through a post-crosslinking reaction. ${ }^{13-15}$ 
Secondly, the reactive mesogen may be physically mixed with the polymer during the film preparation process. ${ }^{16-18}$ Thirdly, a reactive mesogen coating layer may be applied to the aligning film. ${ }^{19,20}$ In the above-mentioned systems, the reactive mesogen on the alignment layer provides strong interactions with LC molecules, such as $\pi-\pi$ stacking interactions or dipole-dipole interactions. Moreover, liquid-crystalline properties of the mesogen would also play a role in its physical guidance towards LC molecules, imparting it with solubility and mobility in LCs. ${ }^{21,22}$ However, the incorporation of reactive mesogens in such photo-reactive materials has always degraded the transparency of the aligning film, despite the high efficiency in improving the aligning ability. Therefore, it is necessary to prepare an alignment polymer substrate with improved properties in terms of both optical transparency and azimuthal anchoring energy.

Conventional PIs usually have a brown colour and strong absorption in the visible light region caused by intermolecular and intramolecular charge-transfer complexes between alternating electron-donor (diamine) and electron-acceptor (dianhydride) moieties. ${ }^{23,24}$ This colour and opacity would ultimately affect the imaging resolution and brightness of LCDs. Hence, it is of practical importance to develop PIs with high optical transparency and strong aligning ability towards LC molecules without sacrificing their excellent thermal and mechanical properties.

One effective way to improve the transparency of PIs is to introduce units with high refractive index into the PI chains. ${ }^{25}$ According to the Lorentz-Lorenz equation, substituents with high molar refraction, low molar volume, or high density, such as aromatic rings, or heavy halogen, sulfur, or metal atoms with high molar refraction, can improve the transparency. ${ }^{26}$ To date, some sulfur-containing PIs with high optical transparency have been applied in optical devices. ${ }^{27}$ In addition, heteroaromatic rings containing a $\mathrm{C}=\mathrm{N}-\mathrm{C}$ unit have also been demonstrated to be effective for enhancing the transparency of polymers, because the $\mathrm{C}-\mathrm{N}=\mathrm{C}$ bond possesses relatively high molar refractivity (4.10) compared to a $\mathrm{C}=\mathrm{C}$ bond $(1.73) .{ }^{28}$ In the present study, use of a monomer including a pyrimidine unit and a sulfur atom is proposed to achieve optical transparency of an aromatic polyimide.

In this work, we have innovatively synthesized a mesogen-copolymerized transparent polyimide as a LC alignment layer by introducing mesogenic units to enhance the azimuthal anchoring energy, selecting a pyrimidine ring and a sulfur atom to improve the transparency. Specifically, cyanobiphenyl mesogen units have been covalently appended to the diamine monomer of the PI, which contained a pyrimidine ring and a sulfur atom. The synthesized functional diamine was copolymerized with 4,4'-diaminodiphenyl ether and 4,4'-oxydiphthalic anhydride to obtain the targeted polyimide (PI-M6). As a candidate as an LC alignment layer for twisted nematic LCD devices, the thermal, mechanical, and optical properties and pretilt angle of the resultant PI have been tested to evaluate its performance. The relationship between the amount of mesogenic groups and the alignment ability of the PI layer has also been investigated.

\section{Experimental}

\subsection{Materials}

Potassium carbonate, sodium hydroxide, methanol, and acetone were purchased from Sinopharm Chemical Reagent Co. (Shanghai, China). 4,6-Diamino-2-mercapto-pyrimidine was purchased from Aladdin (Shanghai, China). 1,6-Dibromohexane and 4-cyano-4'-hydroxybiphenyl were obtained from J\&K Reagent Co. (Beijing, China). 4,4'-Diaminodiphenyl ether (ODA) was purchased from Alfa Aesar (Ward Hill, MA, USA) and purified by recrystallization from ethanol. 4,4'-Oxydiphthalic anhydride (ODPA; >99\%, Suzhou Yacoo Chemical Reagent Corporation, Jiangsu, China) was purified by recrystallization from acetic anhydride and dried under vacuum prior to use. $N, N^{\prime}$-Dimethylformamide (DMF) and $N, N^{\prime}$-dimethylacetamide (DMAc) were distilled under reduced pressure after stirring over calcium hydride for $24 \mathrm{~h}$ and stored over $4 \AA$ molecular sieves before use. A nematic LC of $4^{\prime}$-pentyl-4cyanobiphenyl (5CB) $\left(n_{\mathrm{o}}=1.533, n_{\mathrm{e}}=1.716\right)$ was purchased from Adamas (Shanghai, China).

\subsection{Characterization}

Proton nuclear magnetic resonance $\left({ }^{1} \mathrm{H}\right.$ NMR) spectra were recorded on a Varian Mercury Plus $400 \mathrm{MHz}$ spectrometer (Varian, Palo Alto, CA, USA) using deuterated dimethylsulfoxide $\left(\left[\mathrm{D}_{6}\right] \mathrm{DMSO}\right)$ or chloroform $\left(\mathrm{CDCl}_{3}\right)$ as solvent at room temperature, and the chemical shifts were referenced relative to tetramethylsilane. High-resolution mass spectrometric (HRMS) analysis was performed on a SolariX-70FT-MS Fouriertransform ion cyclotron resonance mass spectrometer (MS) (Bruker Daltonics, Karlsruhe, Germany). Attenuated total reflectance-Fourier-transform infrared (ATR-FTIR) spectra were recorded on a Perkin-Elmer Spectrum 100 FTIR spectrophotometer (Perkin-Elmer, Norwalk, CT, USA). Thermogravimetric analysis (TGA) was performed using a TA TGA Q5000IR instrument (TA Instruments, Inc., New Castle, DE, USA). Samples were heated at a rate of $20{ }^{\circ} \mathrm{C} \mathrm{min}{ }^{-1}$ from room temperature to $700{ }^{\circ} \mathrm{C}$ in nitrogen. Differential scanning calorimetry (DSC) was performed on a TA DSC Q2000 instrument at a heating rate of $10{ }^{\circ} \mathrm{C} \min ^{-1}$ in nitrogen. Absorption spectra of PI films were measured on a Perkin-Elmer Lambda 750 UV/Vis spectrophotometer. Molecular weights and polydispersities were estimated using a Perkin-Elmer Series 200 gel permeation chromatograph (GPC) at $40{ }^{\circ} \mathrm{C}$ equipped with two linear mixed-B columns (Polymer Lab Corporation; pore size: $10 \mu \mathrm{m}$, column size: $300 \times$ $7.5 \mathrm{~mm}$ ) and a refractive index detector. DMF (containing $0.03 \mathrm{~mol} \mathrm{~L}^{-1} \mathrm{LiBr}$ and $0.03 \mathrm{~mol} \mathrm{~L}{ }^{-1} \mathrm{H}_{3} \mathrm{PO}_{4}$ ) and polystyrene were used as the eluent (elution rate: $1.0 \mathrm{~mL} \mathrm{~min}^{-1}$ ) and the calibration standard, respectively. A spin-coating process was executed using a KW-4A spinner from Scientific Instrument Co., Ltd. (Xiamen, China). The rubbing process was performed using a rubbing machine from Tsing Electronics Co. Ltd. (Beijing, China). A polarizing optical microscope (POM) was used to evaluate the alignment behavior of the LCs. The pretilt angles of the LCs were measured by the crystal rotation method using a pretilt angle tester LCT-5016 from Changchun Institute of 
Optics, Fine Mechanics and Physics (Changchun, China), and at least five different points on cells were selected for measurement. The azimuthal anchoring energy was measured by means of the torque balance method. ${ }^{29}$ The electro-optical properties of the cells were measured using an He-Ne laser, a digitized oscilloscope, two crossed polarizers, and a photodetector. The actual twist angle was obtained by rotating the analyzer and polarizer together in steps of $0.1^{\circ}$ and noting the angle of minimum transmittance.

\subsection{Monomer synthesis}

1-Bromo-6-(4-cyanobiphenyl-4'-oxy)hexane. ${ }^{30}$ A mixture of 4cyano-4'-hydroxybiphenyl (4.88 g, $0.025 \mathrm{~mol}), 1,2$-dibromohexane $(24.15 \mathrm{~g}, 0.1 \mathrm{~mol})$, potassium carbonate $(7.01 \mathrm{~g}, 0.05 \mathrm{~mol})$, and acetone was stirred under reflux for $24 \mathrm{~h}$. The reaction mixture was filtered while hot, and the residue was washed with acetone. The acetone was removed under reduced pressure and petroleum ether was added to the concentrated organic extract. The resulting precipitate was collected and dried. The crude product was recrystallized from ethanol. Yield: $7.9 \mathrm{~g}, 88.7 \% .{ }^{1} \mathrm{H}$ NMR (400 MHz, $\left.\mathrm{CDCl}_{3}, \delta(\mathrm{ppm})\right): 7.64(\mathrm{ArH}, 4 \mathrm{H}), 7.55(\mathrm{ArH}, 2 \mathrm{H})$, $7.00(\mathrm{ArH}, 2 \mathrm{H}), 4.04\left(\mathrm{OCH}_{2}, 2 \mathrm{H}\right), 3.43\left(\mathrm{CH}_{2} \mathrm{Br}, 2 \mathrm{H}\right), 1.89\left(\mathrm{CH}_{2}{ }^{-}\right.$ $\left.\mathrm{CH}_{2} \mathrm{Br}, 2 \mathrm{H}\right), 1.83\left(\mathrm{OCH}_{2} \mathrm{CH}_{2}, 2 \mathrm{H}\right), 1.56-1.49\left(\mathrm{OCH}_{2} \mathrm{CH}_{2} \mathrm{C}_{2} \mathrm{H}_{4}^{-}\right.$ $\left.\mathrm{CH}_{2} \mathrm{CH}_{2} \mathrm{Br}, 4 \mathrm{H}\right)$.

2-((6-(4-Cyanobiphenyl-4'-oxy)hexyl)thio)pyrimidine-4,6diamine (M6). ${ }^{31}$ In a flask, 4,6-diamino-2-mercapto-pyrimidine (1.42 g, $10 \mathrm{mmol})$, aqueous $\mathrm{NaOH}(0.25 \mathrm{~m}, 42 \mathrm{~mL})$, and methanol $(40 \mathrm{~mL})$ were combined. The reaction mixture was stirred with a magnetic stirrer at room temperature for $4 \mathrm{~h}$ to form the corresponding sodium thiolate salt. The solvent was then evaporated under vacuum. The dry sodium thiolate salt was dissolved in dry DMF (60 mL), and 1-bromo-6-(4-cyanobiphenyl4'-oxy)hexane (3.92 g, $11 \mathrm{mmol}, 1.1$ equiv) was added. The resulting solution was stirred at room temperature for $18 \mathrm{~h}$. The progress of the reaction was monitored by thin-layer chromatography $\left(\mathrm{CH}_{2} \mathrm{Cl}_{2} / \mathrm{MeOH}, 18: 1\right)$. After completion, the reaction was quenched with water, and the mixture was extracted with $\mathrm{CH}_{2} \mathrm{Cl}_{2}$. The organic layer was collected and concentrated. The crude material was purified by flash chromatography $\left(\mathrm{CH}_{2} \mathrm{Cl}_{2} /\right.$ $\mathrm{MeOH}, 8: 1)$ to give the desired product. The product was obtained as a white solid in $83.3 \%$ yield $(3.5 \mathrm{~g}), \mathrm{mp}$ : $188-189{ }^{\circ} \mathrm{C}$. FTIR (KBr, pellet), $\mathrm{cm}^{-1}$ : $3480(\mathrm{~N}-\mathrm{H}) ; 2225$ (-CN). ${ }^{1} \mathrm{H}$ NMR (400 MHz, [D $\mathrm{D}_{6}$ DMSO, $\left.\delta(\mathrm{ppm})\right): 7.83(\mathrm{ArH}, 4 \mathrm{H}), 7.67$ (ArH, 2H), 7.02 (ArH, $2 \mathrm{H}), 6.01\left(\mathrm{NH}_{2}, 4 \mathrm{H}\right), 5.13(\mathrm{ArH}, 1 \mathrm{H}), 4.00\left(\mathrm{OCH}_{2}, 2 \mathrm{H}\right), 2.95$ $\left(\mathrm{SCH}_{2}, 2 \mathrm{H}\right), 1.71\left(\mathrm{OCH}_{2} \mathrm{CH}_{2}, 2 \mathrm{H}\right), 1.59\left(\mathrm{SCH}_{2} \mathrm{CH}_{2}, 2 \mathrm{H}\right), 1.43$ $\left(\mathrm{OCH}_{2} \mathrm{CH}_{2} \mathrm{C}_{2} \mathrm{H}_{4} \mathrm{CH}_{2} \mathrm{CH}_{2} \mathrm{~S}, 4 \mathrm{H}\right)$. High-resolution MS (ESI, $\mathrm{m} / \mathrm{z}$ ): $\left[\mathrm{M}^{+}\right]$calcd for $\mathrm{C}_{23} \mathrm{H}_{25} \mathrm{~N}_{5} \mathrm{OS}$ : 419.18; found: 419.19 .

The chemical structure of M6 was confirmed with FTIR, ${ }^{1} \mathrm{H}$ NMR, and MS (data in the Experimental section). All of the spectroscopic data obtained were consistent with the proposed structure (Scheme 1). The respective ${ }^{1} \mathrm{H}$ NMR peaks of M6 could be unequivocally assigned (Fig. 1).

\subsection{Preparation of PI-M6}

As the content of M6 reached 50\%, the film became brittle after thermal treatment due to the rigidity of the pyrimidine
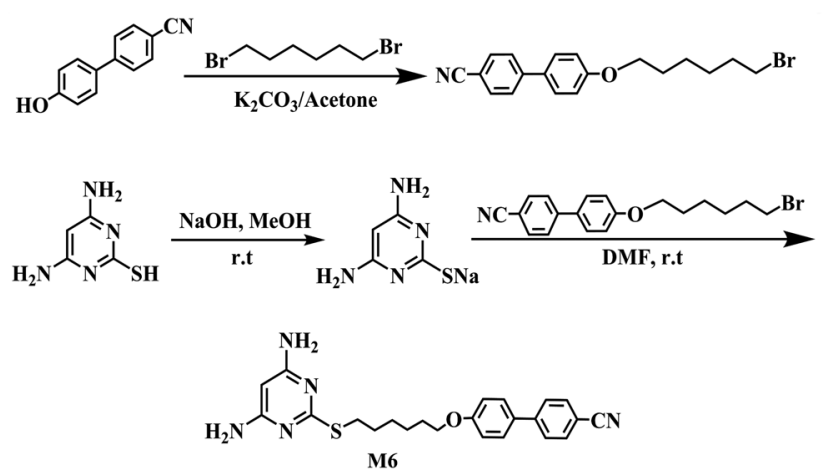

Scheme 1 Synthetic route to the diamine (M6).

unit. In this work, M6/ODA/ODPA mixtures with molar ratios of $0-0.3: 1-0.7: 1$ were applied to prepare PI $_{x}$-M6 (Scheme 2). Here, " $x$ " denotes the molar ratio of M6 to ODA in the polymer. The synthesis of PI is exemplified by the case of $\mathrm{PI}_{1}-\mathbf{M 6}$. M6 (0.4192 g, $1 \mathrm{mmol})$ and ODA (1.8022 g, $9 \mathrm{mmol})$ were dissolved in DMAc (16.12 g) in a $100 \mathrm{~mL}$ three-necked flask fitted with a mechanical stirrer and a nitrogen inlet. After complete dissolution of the diamines, the flask was cooled to $5{ }^{\circ} \mathrm{C}$ in an ice bath. A solution of ODPA $(3.1022 \mathrm{~g}, 10 \mathrm{mmol})$ in DMAc $(5.31 \mathrm{~g})$ was added, and the resultant mixture was stirred under a gentle flow of dry nitrogen. After $10 \mathrm{~h}$, a viscous, homogeneous solution of polyamic acid (PAA) had formed.

A $\mathrm{PI}_{1}$-M6 film was obtained by thermal imidization of the PAA solution cast on a clean glass plate. This involved a preheating program $\left(70{ }^{\circ} \mathrm{C} / 1 \mathrm{~h}, 90^{\circ} \mathrm{C} / 2 \mathrm{~h}, 110^{\circ} \mathrm{C} / 1 \mathrm{~h}\right)$ followed by the imidization procedure $\left(200{ }^{\circ} \mathrm{C} / 2 \mathrm{~h}, 220{ }^{\circ} \mathrm{C} / 2 \mathrm{~h}, 250{ }^{\circ} \mathrm{C} / 2 \mathrm{~h}\right)$ to produce a fully imidized $\mathrm{PI}_{1}-\mathbf{M} 6$ film. The $\mathrm{PI}_{1}-\mathbf{M} 6$ film was exfoliated by placing the glass plate in hot water. The other PIM6 films were prepared analogously.

\subsection{Fabrication of the LC cells}

Precursor films of the alignment layers were fabricated by spincoating PAA solutions ( $8 \mathrm{wt} \%$ in DMAc), at rotation speeds of $900 \mathrm{rpm}$ for $10 \mathrm{~s}$ and $2800 \mathrm{rpm}$ for $30 \mathrm{~s}$, on clean indium tin oxide (ITO)-coated glass substrates of dimensions $25 \times 25 \times 0.5$ $\mathrm{mm}$. The precursor films were baked at $100{ }^{\circ} \mathrm{C}$ for $30 \mathrm{~min}$ to

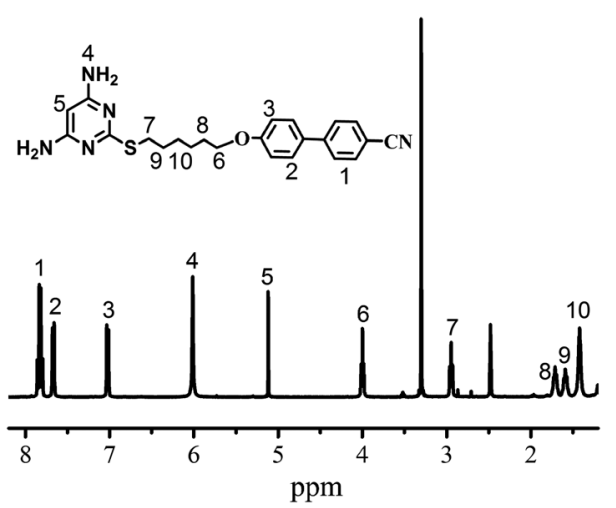

Fig. $1{ }^{1} \mathrm{H}$ NMR spectra of the M6 in $\left[D_{6}\right]$ DMSO. 


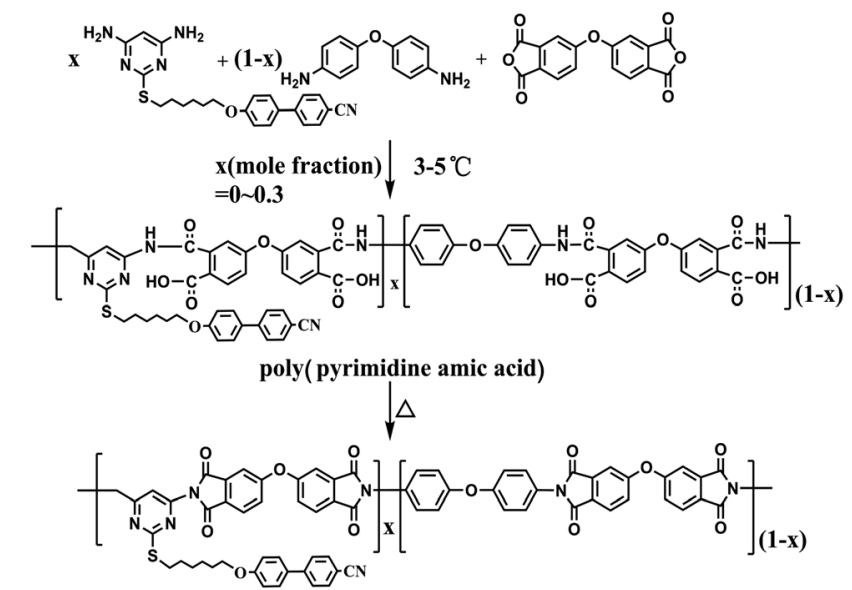

Scheme 2 Synthetic route to all of the PI-M6

evaporate the solvent. The films were then thermally imidized at $200{ }^{\circ} \mathrm{C}$ for $2 \mathrm{~h}$ followed by $250^{\circ} \mathrm{C}$ for $1 \mathrm{~h}$. The thickness of the alignment layer was about $500 \mathrm{~nm}$ by means of a film thickness measuring instruments. The prepared PI-M6 films on glass were subsequently unidirectionally rubbed using a velvet-cloth rubbing machine at a rotation speed of the rubbing drum of $1000 \mathrm{rpm}$, a translation speed of the film of $20 \mathrm{~mm} \mathrm{~s}^{-1}$, and a pile impression of $0.4 \mathrm{~mm}$.

Each LC cell was assembled by using two pieces of rubbed glass substrate in an anti-parallel rubbing direction. The cell thickness was controlled at $5 \mu \mathrm{m}$ by thick spacers. A nematic LC (5CB) was injected into the cell at $50{ }^{\circ} \mathrm{C}$ by capillary force, and then the cell was sealed with epoxy glue. The LC cells were then treated for $20 \mathrm{~min}$ at $50{ }^{\circ} \mathrm{C}$ to attain the isotropic phase of the LC, and subsequently cooled to room temperature. The pretilt angles were measured using a crystal rotation method. The measurement method has been described in the ESI. $\dagger$ The alignment behavior of the LC molecules was examined with a polarized optical microscope.

\subsection{Measurements of the azimuthal anchoring energy}

To measure the azimuthal anchoring energy on PI-M6 films, as a characteristic of the LC alignment, twist LC cells constructed from two substrates were prepared. The thickness of the twist LC cells, $d$, was controlled in the region of 13-14 $\mu \mathrm{m}$ by spacers. LC cells were filled with $5 \mathrm{CB}$ in its isotropic phase and slowly cooled to room temperature. The experimental set-up for measurements of azimuthal anchoring energy $(W)$ is shown schematically in Fig. 2. $W$ was calculated by the following equation: ${ }^{29}$

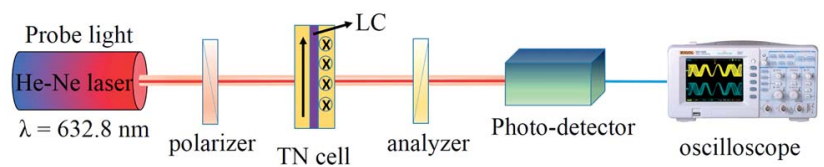

Fig. 2 Experimental set-up for measuring azimuthal anchoring energy.

$$
W=\frac{2 K_{22} \varphi}{d \sin (\Phi-\varphi)}
$$

where $d$ is the thickness of the LC cell, $\Phi$ is the deviation angle between the easy axes of the two substrates, $K_{22}$ is the twist elastic constant of the LC, and $\varphi$ is the actual twist angle in the TN cell.

\subsection{Measurement of surface adhesion force of PI-M6 films to LCs}

The adhesion force is defined as the force required to lift an LC from a PI alignment film. The adhesion force of PI-M6 films to LCs is a vitally important characteristic of anchoring energy, which has never been quantified due to the lack of a measurement method. In this study, the adhesion force was measured by using a super-sensitive microelectro-mechanical balance system (self-regulating DCAT 11, Dataphysics). The adhesion values are the averages for four samples. A PI-M6 film of fixed size $(15 \times 5 \times 0.02 \mathrm{~mm})$ was anchored by a clip. The LC in its liquid state was introduced into the container, which was then placed on a balance table. The LC solution was moved upwards at a rate of $1 \mathrm{~mm} \mathrm{~s}^{-1}$ until it immersed the PI-M6 film at a depth of $5 \mathrm{~mm}$. Then, the LC solution was moved down at a rate of $0.1 \mathrm{~mm} \mathrm{~s}^{-1}$. Throughout this process, the adhesion force was tested and recorded.

\section{Results and discussion}

\subsection{Synthesis and characterization of PI-M6 films}

To investigate the effect of the functional diamine on the transparency and surface anchoring strength of alignment films, pyrimidine-containing PI-M6 samples were synthesized by reacting of M6/ODA/ODPA at molar ratios of $0-0.3: 1-0.7: 1$ according to a conventional two-step procedure, as shown in Scheme 2. The molecular weights of the PAAs were measured by GPC and are summarized in Table 1. The molecular weights of PAAs decreased with increasing amount of pyrimidyl diamine. This can be attributed to the relatively low reactivity of the functional diamine, due to its steric hindrance.

ATR-FTIR spectra were recorded to confirm the successful synthesis of PI-M6, and are shown in Fig. 3. The characteristic IR bands of PAA (the carbonyl absorption at $\nu=1651 \mathrm{~cm}^{-1}$ and the broad carboxylic acid absorption at $\nu=3387 \mathrm{~cm}^{-1}$ ) had clearly disappeared in the ATR-FTIR spectra of all of the PI films, whereas strong bands at $\nu=1780$ and $1714 \mathrm{~cm}^{-1}$, attributable to the $-\mathrm{C}=\mathrm{O}$ asymmetric and symmetric stretching vibration of imide rings, were observed for all of the PI-M6 samples, indicating the successful conversion of PAAs into PIs. In addition, stretching vibrations of the pyrimidine ring at $\nu=1563 \mathrm{~cm}^{-1}$ and the $-\mathrm{CN}$ group at $\nu=2225 \mathrm{~cm}^{-1}$ were observed, and their intensity increased with increasing molar ratio of M6/ODA in the PI-M6 backbones, further confirming that the functional monomer had been co-polymerized into PI chains. 
Table 1 Thermal performances of PI-M6 and molecular weights of PAAs

\begin{tabular}{lllllrr}
\hline PIs & $T_{\mathrm{g}}{ }^{a}\left({ }^{\circ} \mathrm{C}\right)$ & $T_{\mathrm{d} 5}{ }^{b}\left({ }^{\circ} \mathrm{C}\right)$ & $T_{\mathrm{d} 10}{ }^{b}\left({ }^{\circ} \mathrm{C}\right)$ & $R_{\mathrm{w}}{ }^{c}(\%)$ & $M_{\mathrm{n}}{ }^{d}\left(\times 10^{-4}\right)$ & $M_{\mathrm{w}}{ }^{d}\left(\times 10^{-4}\right)$ \\
\hline $\mathrm{PI}_{0}$-M6 & 244.6 & 512.1 & 550.5 & 54.7 & 12.09 & 23.15 \\
$\mathrm{PI}_{1}$-M6 & 228.5 & 436.2 & 484.4 & 56.6 & 3.29 & 7.07 \\
$\mathrm{PI}_{2}$-M6 & 208.6 & 427.6 & 444.8 & 51.9 & 2.61 & 2.91 \\
$\mathrm{PI}_{3}$-M6 & 194.1 & 420.3 & 436.1 & 47.3 & 1.77 & 2.14 \\
\end{tabular}

${ }^{a}$ From the second DSC heating scan at a heating rate of $20{ }^{\circ} \mathrm{C} \mathrm{min}^{-1}$ in nitrogen. ${ }^{b}$ Temperatures corresponding to $5 \%$ and $10 \%$ weight losses by thermogravimetry at a heating rate of $20^{\circ} \mathrm{C} \mathrm{min}^{-1}$ in nitrogen. ${ }^{c}$ Residual weight $\%$ at $700{ }^{\circ} \mathrm{C} .{ }^{d}$ Measured by GPC in DMF; polystyrene was used as a standard.

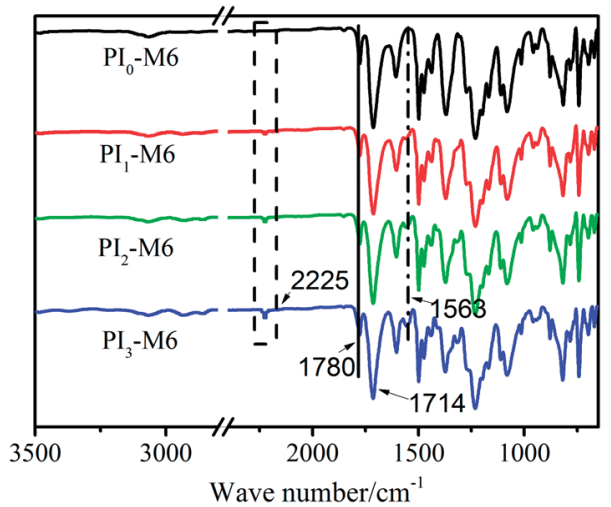

Fig. 3 ATR FTIR spectra of PI-M6 films.

\subsection{Properties of PI-M6 films}

Thermal properties. The thermal properties of the PI-M6 films were evaluated by DSC as well as TGA under a nitrogen atmosphere, and the thermal data are summarized in Table 1. Fig. 4(a) shows DSC curves for the second heating scans of the PI-M6 films. The $T_{\mathrm{g}}$ values for all of the PI-M6 samples were in the range $194-245^{\circ} \mathrm{C}$. As expected, the $T_{\mathrm{g}}$ values of these PI-M6 decreased with increasing amount of pyrimidyl diamine, which can be attributed to its long side chains. The long side chains resulted in loose chain packing and weak intermolecular interactions, such that the free volume in the polymer segments increased, thereby reducing $T_{\mathrm{g}}$ of the PI-M6. ${ }^{32}$

Fig. 4(b) shows TGA curves obtained under nitrogen. The decomposition temperatures corresponding to $5 \%$ and $10 \%$ weight losses $\left(T_{\mathrm{d} 5}\right.$ and $\left.T_{\mathrm{d} 10}\right)$ and the residual weights at $700{ }^{\circ} \mathrm{C}$ $\left(R_{\mathrm{W}}\right)$ are summarized in Table 1 . In contrast to the $\mathrm{PI}_{0}-\mathbf{M 6}$ film, the films with mesogenic side chains showed two-step weight loss behavior. The weight loss associated with the first step
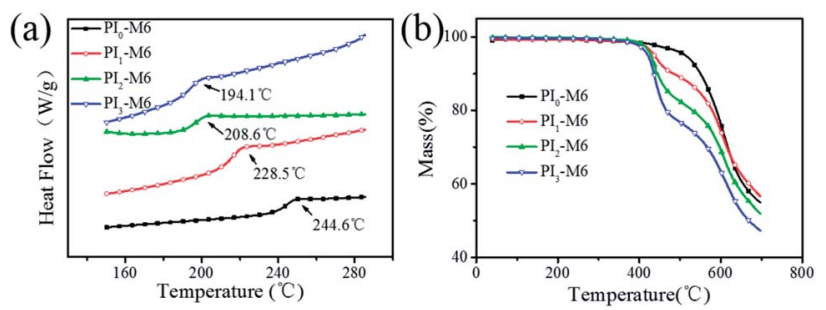

Fig. 4 DSC curves (a) and TGA curves in nitrogen (b) of the PI-M6. implies that the side chains degrade at $c a .410{ }^{\circ} \mathrm{C}$. The weight loss associated with the second step occurred in the same temperature range as the degradation of $\mathrm{PI}_{0}-\mathbf{M 6}$, and can thus be attributed to degradation of the PI-M6 main chain. Although introduction of the functional diamine was somewhat detrimental to the thermal stability of the PI film, all of the obtained PI-M6 films still presented $T_{\mathrm{d} 5}>420{ }^{\circ} \mathrm{C}$ and $T_{\mathrm{d} 10}>436{ }^{\circ} \mathrm{C}$, with char yields $>47 \%$ after pyrolysis at $700{ }^{\circ} \mathrm{C}$. These properties are sufficient to meet the technical requirements of LCDs.

Optical properties. Optical transparency of PI films is very important for their application in optoelectronic devices, such as organic thin-film transistors, photonic materials, LCD device materials, and so on. All of the prepared PI-M6 films were optically transparent, with thicknesses of $\sim 20 \mu \mathrm{m}$ (Fig. 5(a)). Furthermore, the films could be bent, indicating flexibility and good mechanical properties. The salient mechanical properties, including tensile strength, tensile modulus, and elongation at break, are summarized in Table S1. $\dagger$ The UV/Vis spectra are shown in Fig. 5(b). All of the PI-M6 films showed good optical transparency in the wavelength range 450-700 nm. $\mathrm{PI}_{1}-\mathbf{M 6}, \mathrm{PI}_{2}-\mathbf{M 6}$, and $\mathrm{PI}_{3}-\mathbf{M 6}$ even exhibited much higher transparency (above 86\%) than $\mathrm{PI}_{0}-\mathbf{M 6}$ (about $80 \%$ ), indicating that the pyrimidine rings and sulfur atoms with high molar refractivity are indeed beneficial for improving the optical transparency of PIs.

\subsection{Properties of LC cells}

A POM was used to evaluate the alignment behavior of the LCs by placing LC cells between its two crossed polarizers. For the $\mathrm{PI}_{2}$-M6 cell (Fig. 6), the light field showed alternating changes between dark and bright under the crossed polarizers upon rotation of the cell over a period of $90^{\circ}$, explicitly indicating that
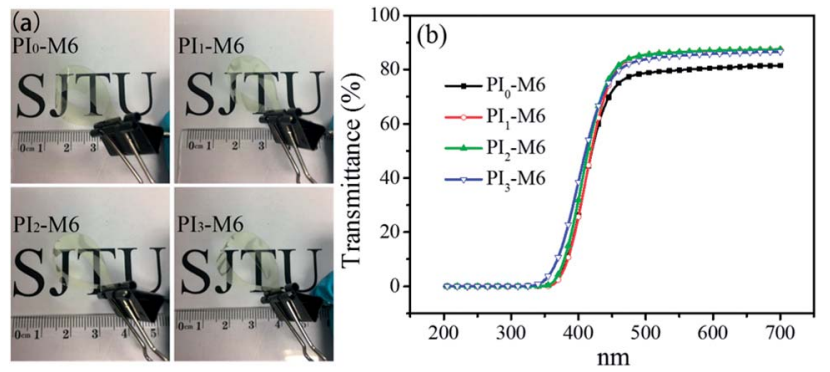

Fig. 5 (a) Optical photographs and (b) optical transmission spectra of PI-M6 films. 

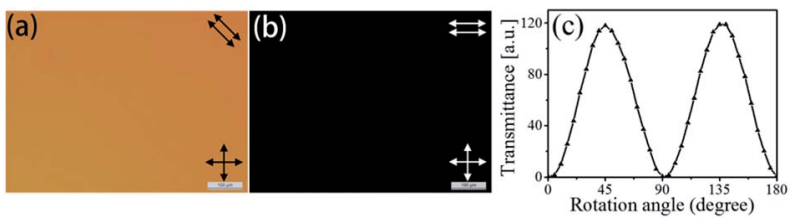

Fig. 6 POM images of $\mathrm{LC}$ alignment on the $\mathrm{Pl}_{2}-\mathrm{M} 6$ substrate taken between crossed polarizers: (a) the rubbing direction at $45^{\circ}$ to each polarizer axes; (b) the rubbing direction parallel to one of the polarizer axes; (c) optical transmittance of LC cells as a function of the rotation angle of the cell between crossed polarizers.

homogeneous and stable LC alignment was achieved on the rubbed orientating film. All of the PI-M6 films exhibited good alignment performance towards LC molecules in the LC cell.

Pretilt angle. It is generally believed that the length of an alkyl side chain is directly related to the pretilt angle of the polyimide film. Therefore, we investigated the effect of the content of M6 diamine on the pretilt angle, and the results are shown in Fig. 7. It can be seen that the pretilt angle of the LC cell was only $0.2^{\circ}$ for the rubbed polyimide film without $\mathbf{M 6}\left(\mathrm{PI}_{0}-\mathbf{M 6}\right)$, and then increased monotonically from $0.2^{\circ}$ to $4.2^{\circ}$, with an increase in the molar fraction of $\mathbf{M 6}$ with respect to total diamine from 0 to $30 \%$. The $\mathrm{PI}_{3}$-M6 film with the highest content of M6 showed the largest pretilt angle, which can be attributed to the ordered stacking density of long alkyl side chains on the polyimide surface. ${ }^{33}$ Due to the similar molecular structure of the polyimide side chains and liquid-crystal molecules, the orientation direction of the rubbed mesogen side chains was efficiently transmitted to the liquid-crystal molecules. That is to say, mesogen side-chain aligned liquid crystals could be obtained.

Azimuthal anchoring energy. To reveal the relationship between the surface anchoring strength of the rubbed PI films and the density of the mesogen side chains, the dependence of the real twist angle $\varphi$ of LC cells on the density of side chains of polyimide was measured, and the results are listed in Table 2. The deviation angle between the easy axes of the two substrates is $90^{\circ} . K_{22}$ of $5 \mathrm{CB}$ is $4.22 \times 10^{-12} \mathrm{~N}$. The $W$ of PI-M6 could be calculated according to eqn (1), and the results showed that the azimuthal anchoring energy distinctly increased with increasing content of the mesogen side chains. The $W$ of $\mathrm{PI}_{3}-\mathbf{M 6}$

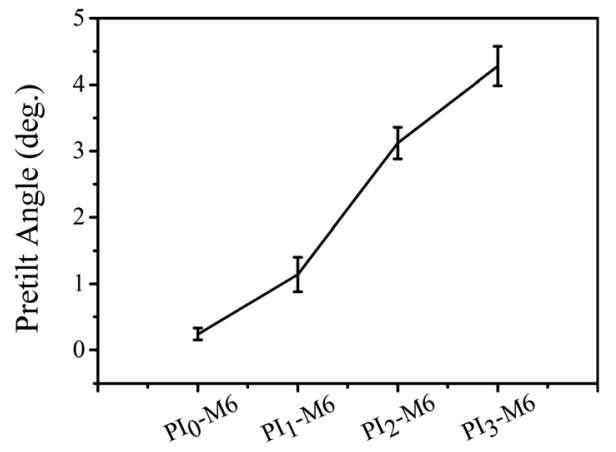

Fig. 7 Pretilt angles of LC cells fabricated with PI-M6 as LC alignment layers.
Table 2 Calculated results for the azimuthal anchoring energies

\begin{tabular}{llll}
\hline PIs & $d(\mu \mathrm{m})$ & $\varphi(\mathrm{deg})$ & $W\left(\mathrm{~J} \mathrm{~m}^{-2}\right)$ \\
\hline $\mathrm{PI}_{0}$-M6 & 13.73 & 87.95 & $2.51 \times 10^{-5}$ \\
$\mathrm{PI}_{1}$-M6 & 13.73 & 88.35 & $3.18 \times 10^{-5}$ \\
$\mathrm{PI}_{2}$-M6 & 13.96 & 88.92 & $4.77 \times 10^{-5}$ \\
$\mathrm{PI}_{3}$-M6 & 13.61 & 89.01 & $5.72 \times 10^{-5}$
\end{tabular}

was more than twice that of $\mathrm{PI}_{0}-\mathbf{M 6}$. This may be attributed to the strong interaction between the side chain and the LC molecule, which included dipole-dipole and $\pi-\pi$ interactions between the biphenyl groups of 5CB molecules and the mesogen side chains, and the van der Waals forces between the alkoxy spacer of the mesogen groups and the flexible alkoxy tail of the 5CB molecule. ${ }^{34}$

To determine the mechanism underpinning the enhanced anchoring energy, the adhesion force between the PI-M6 film and an LC was measured on the as-prepared DCAT 11 equipped with a super-sensitive microelectro-mechanical balance system. The LC solution was introduced into a container, which was placed on the plate of the balance system (Fig. 8(a)). A PI-M6 film was held above the solution, and the balance system was initialized to zero. The LC solution was then lifted up to come into contact with the film (the force remained at zero during this Process 1). Due to the strong adhesion between the LC solution and the PI-M6 film, the force balance increased sharply when the solution touched the film. The LC was further moved upwards until the targeted immersion depth of $5 \mathrm{~mm}$ was

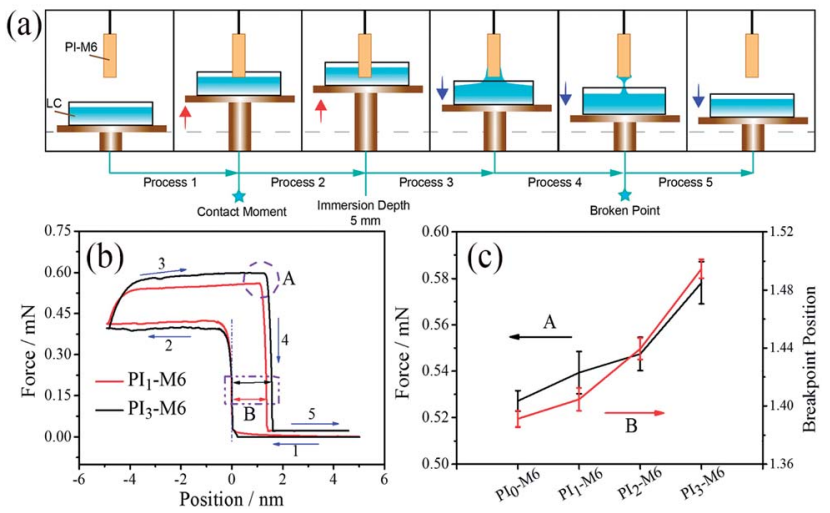

Fig. 8 (a) Experimental set-up for measuring of surface adhesion force of PI-M6 films to LC. Process 1, the LC solution moved upward to film and the mass of film was set to zero as control. Contributing to the high affinity, LC would climb onto the PI-M6 film surface at the moment of the LC solution touching films and leaded to a sharp increase of adhesion force. Process 2, the LC solution continue to move upward until immersing $5 \mathrm{~mm}$ of PI-M6 film. Process 3, LC solution move down. During this process, the adhesion force increased because the increasing contact area and the decrease of buoyancy. At the end of process 3, the LC liquid was stretched and present a strongest adhesion force. Process 4, the connection between LC and PI-M6 film was broken. The LC moved down away from the surface. (b) Force-distance curves through entire immersion process of the film in and off LC solution. (c) The maximum force and film breakpoint position of PI-M6. 
reached (Process 2). Thereafter, the adhesion force between the LC solution and the PI-M6 film was measured as the former was slowly lowered (Process 3). As illustrated in Fig. 8(b) for $\mathrm{PI}_{3}-\mathbf{M 6}$, the adhesion force gradually increased due to the decrease of buoyancy. Moreover, the LC solution formed a stretched liquid layer after returning to the original start point. At the end of Process 3, the LC layer remained stretched and presented a strong adhesion force. The force reached a maximum at the point of separation from the LC layer. For different PI-M6 films, the largest adhesion force and highest point of separation indicated the maximum adhesion force between the film and the LC.

For the unmodified PI film without mesogen groups, the maximum force was $0.53 \mathrm{mN}$, and the maximum point of separation was at $1.39 \mathrm{~mm}$. After co-polymerization of the diamine with mesogen side chains, both the force and point of separation increased sharply (Fig. 8(c)). As mentioned above, the mesogen molecules could interact with 5CB through dipoledipole and $\pi-\pi$ interactions. As a result, the adhesion force would be improved with increasing content of mesogen groups. The adhesion force on $\mathrm{PI}_{3}$-M6 increased to $0.58 \mathrm{mN}$, and the point of separation was extended to $1.49 \mathrm{~mm}$. The enhanced adhesion force indicated that the introduction of mesogen groups can directly improve the interactions between the LC and the alignment layer PI-M6. Such interactions would ultimately influence the capacity of the films to guide LC orientation, which can be termed anchoring energy.

\section{Conclusions}

In conclusion, based on mesogen groups aligning liquid-crystal molecules, a novel functional diamine containing pyrimidine and mesogen groups has been synthesized. This diamine has been co-polymerized with ODA and ODPA. The resultant PI-M6 films showed good thermal stability and excellent mechanical properties. Most of these PI-M6 films showed good optical transparency, with transmittances of up to $86 \%$ in the wavelength range 450-700 nm. Rubbed PI-M6 provides a tunable pretilt angle for aligned LC molecules thereon. Moreover, the azimuthal anchoring strength of $\mathrm{PI}_{3}$-M6 was improved more than 2.2-fold compared with that of $\mathrm{PI}_{0}$-M6. Therefore, such PIM6 films could be suitable candidates for applications as alignment layers in LCD devices.

\section{Conflicts of interest}

The authors declare no competing financial interests.

\section{Acknowledgements}

The authors are grateful for financial support from the National Science Foundation of China (51733007 and 21574081) and 973 Projects (2014CB643605).

\section{References}

1 Q. Gong, S. M. Gong, H. Zhang, L. L. Liu and Y. H. Wang, Synthesis of a novel polyimide used as liquid crystal vertical alignment layers, RSC Adv., 2015, 5, 57245-57253.

2 B. J. Mun, K. W. Park, J. H. Baek, B. K. Kim, H. C. Choi, C. W. Kim, B. Kang, S. H. Lee and G. D. Lee, Timesequential ultraviolet exposure to alignment layers embedded with reactive mesogen for high-speed in-plane switching liquid crystal cell, IEEE Trans. Electron Devices, 2016, 63, 4326-4330.

3 S. Y. Kim, H. G. Park, M. J. Cho, H. C. Jeong and D. S. Seo, Nanocrystalline LaYSrO films for liquid-crystal alignment via a solution process, Liq. Cryst., 2014, 41, 940-945.

4 H. Yeo, M. Goh, B. C. Ku and N. H. You, Synthesis and characterization of highly-fluorinated colorless polyimides derived from 4,4'-((perfluoro-[1,1'-biphenyl]-4,4'-diyl) bis(oxy))bis(2,6-dimethylaniline) and aromatic dianhydrides, Polymer, 2015, 76, 280-286.

5 Y. C. Ding, H. Q. Hou, Y. Zhao, Z. T. Zhu and H. Fong, Electrospun polyimide nanofibers and their applications, Prog. Polym. Sci., 2016, 61, 67-103.

6 S. Wurth, M. Capogrosso, S. Raspopovic, J. Gandar, G. Federici, N. Kinany, A. Cutrone, A. Piersigilli, N. Pavlova, R. Guiet, G. Taverni, J. Rigosa, P. Shkorbatova, X. Navarro, Q. Barraud, G. Courtine and S. Micera, Long-term usability and bio-integration of polyimide-based intra-neural stimulating electrodes, Biomaterials, 2017, 122, 114-129.

7 T. Yoon, N. Chapman, C. C. Nguyen and B. L. Lucht, Electrochemical reactivity of polyimide and feasibility as a conductive binder for silicon negative electrodes, $J$. Mater. Sci., 2017, 52, 3613-3621.

8 Y. Q. Fang, J. Wang, Q. Zhang, Y. Zeng and Y. H. Wang, Synthesis of soluble polyimides for vertical alignment of liquid crystal via one-step method, Eur. Polym. J., 2010, 46, 1163-1167.

9 P. K. Son and S. W. Choi, Study on the relation between rubbing conditions and physical parameters of polyimide, Mol. Cryst. Liq. Cryst., 2011, 546, 26-33.

10 H. Lai, X. Y. Liu, L. Qin, M. Li and Y. Gu, Preparation of vertical alignment layers by blending polyimide precursors with and without side chains, Liq. Cryst., 2009, 36, 173-178.

11 J. Wang, L. Wang, Y. Zeng, Y. Q. Fang, Q. Zhang and Y. H. Wang, A study of the transition of liquid-crystal alignment from homeotropic to planar on a polyimide layer, Liq. Cryst., 2010, 37, 271-278.

12 G. Cheng, B. Spraul, D. Smith and D. Perahia, Semi fluorinated polymers as surface energy controlled layers for liquid crystal alignment, RSC Adv., 2016, 6, 69412-69420.

13 S. G. Kim, S. M. Kim, Y. S. Kim, H. K. Lee, S. H. Lee, G. D. Lee, J. J. Lyu and K. H. Kim, Stabilization of the liquid crystal director in the patterned vertical alignment mode through formation of pretilt angle by reactive mesogen, Appl. Phys. Lett., 2007, 90, 261910-261913.

14 Y. J. Lim, I. H. Jeong, H. S. Kang, S. Kundu, M. H. Lee and S. H. Lee, Reduction of the residual DC in the 
photoaligned twisted nematic liquid crystal display using polymerized reactive mesogen, Appl. Phys. Express, 2012, 5, 081701-081703.

15 V. Finnemeyer, D. Bryant, R. Reich, S. Berry, C. Bozler, O. Yaroshchuk, L. Lu and P. Bos, Versatile alignment layer method for new types of liquid crystal photonic devices, $J$. Appl. Phys., 2015, 118, 034501-034508.

16 S. W. Oh, J. H. Park and T. H. Yoon, Near-zero pretilt alignment of liquid crystals using polyimide films doped with UV-curable polymer, Opt. Express, 2015, 23, 1044-1051.

17 K. H. Kim, J. I. Baek, B. H. Cheong, H. Y. Choi, S. T. Shin, J. C. Kim and T. H. Yoon, Pretilt angle control and multidomain alignment of liquid crystals by using polyimide mixed with liquid crystalline prepolymer, Appl. Phys. Lett., 2010, 96, 2135071-2135073.

18 Q. Guo, A. K. Srivastava, V. G. Chigrinov and H. S. Kwok, Polymer and azo-dye composite: a photo-alignment layer for liquid crystals, Liq. Cryst., 2014, 41, 1465-1472.

19 Y. K. Moon, Y. J. Lee, S. I. Jo, Y. Kim, J. U. Heo, J. H. Baek, S. G. Kang, C. J. Yu and J. H. Kim, Effects of surface modification with reactive mesogen on the anchoring strength of liquid crystals, J. Appl. Phys., 2013, 113, 2345041-2345044.

20 O. Yaroshchuk, V. Kyrychenko, D. Tao, V. Chigrinov, H. S. Kwok, H. Hasebe and H. Takatsu, Stabilization of liquid crystal photoaligning layers by reactive mesogens, Appl. Phys. Lett., 2009, 95, 0219021-02190213.

21 Y. Kim, Y. J. Lee, J. H. Baek, C. J. Yu and J. H. Kim, Dependence of planar alignment layer upon enhancement of azimuthal anchoring energy by reactive mesogens, Jpn. J. Appl. Phys., 2014, 54, 0117011-0117015.

22 M. C. Tseng, O. Yaroshchuk, T. Bidna, A. K. Srivastava, V. Chigrinov and H. S. Kwok, Strengthening of liquid crystal photoalignment on azo dye films: passivation by reactive mesogens, $R S C A d v .$, 2016, 6, 48181-48188.

23 M. J. Banach, R. H. Friend and H. Sirringhaus, Influence of the molecular weight on the thermotropic alignment of thin liquid crystalline polyfluorene copolymer films, Macromolecules, 2003, 36, 2838-2844.

24 S. Ando, T. Matsuura and S. Sasaki, Coloration of aromatic polyimides and electronic properties of their source materials, Polym. J., 1997, 29, 69-76.

25 Y. Wang, Synthesis and characterization of novel polyimides derived from 2,4-bis(4-aminophenoxy)pyrimidine, High Perform. Polym., 2014, 26, 978-985.
26 N. H. You, Y. Nakamura, Y. Suzuki, T. Higashihara, S. Ando and M. Ueda, Synthesis of highly refractive polyimides derived from 3,6-bis(4-aminophenylenesulfanyl)pyridazine and 4,6-bis(4-aminophenylenesulfanyl)pyrimidine, J. Polym. Sci., Part A: Polym. Chem., 2009, 47, 4886-4894.

27 K. Nakabayashi, T. Imai, M. C. Fu, S. Ando, T. Higashihara and M. Ueda, Synthesis and characterization of poly(phenylene thioether)s containing pyrimidine units exhibiting high transparency, high refractive indices, and low birefringence, J. Mater. Chem. C, 2015, 3, 7081-7087.

28 C. B. Wang, Y. Guan, D. B. Tian, G. D. Dang, D. M. Wang, C. H. Chen and H. W. Zhou, Highly transparent polyimides derived from 2-phenyl-4,6-bis(4-aminophenoxy) pyrimidine and 1,3-bis(5-amino-2-pyridinoxy)benzene: preparation, characterization, and optical properties, $R S C$ $A d v .$, 2015, 5, 103246-103254.

29 Y. Zhou, Z. He and S. Sato, Generalized relation theory of torque balance method for azimuthal anchoring measurements, Jpn. J. Appl. Phys., 1999, 38, 4857-4858.

30 Z. T. Nagy, B. Heinrich, D. Guillon, J. Tomczyk, J. Stumpe and B. Donnio, Heterolithic azobenzene-containing supermolecular tripedal liquid crystals self-organizing into highly segregated bilayered smectic phases, J. Mater. Chem., 2012, 22, 18614-18622.

31 F. Q. Tong, Z. Chen, X. M. Lu and Q. H. Lu, Thermostable birefringent copolyimide films based on azobenzenecontaining pyrimidine diamines, J. Mater. Chem. C, 2017, 5, 10375-10382.

32 S. L. Xia, L. F. Yi, Z. Sun and Y. H. Wang, The effect of phthalimide side chains on the thermal stability and rubbing resistance of polyimide used as a liquid crystal vertical alignment layer, J. Polym. Res., 2013, 20, 219.

33 S. L. Xia, Z. Sun, L. F. Yi and Y. H. Wang, Synthesis of soluble polyimide derived from novel naphthalene diamines for liquid crystal alignment layers and a preliminary study on the mechanism of imidization, RSC Adv., 2013, 3, 1466114670.

34 S. I. Kim, M. Ree, T. J. Shin and J. C. Jung, Synthesis of new aromatic polyimides with various side chains containing a biphenyl mesogen unit and their abilities to control liquid-crystal alignments on the rubbed surface, J. Polym. Sci., Part A: Polym. Chem., 1999, 37, 2909-2921. 